\title{
Lipid-laden dendritic cells fail to function
}

\author{
Philip C Calder ${ }^{1}$ \\ ${ }^{1}$ Institute of Human Nutrition and Developmental Origins of Health and Disease Division, School of Medicine, University of South- \\ ampton, Institute of Developmental Sciences Building, MP887 Southampton General Hospital, Tremona Road, Southampton SO16 \\ $6 Y D$, United Kingdom \\ Cell Research (2010) 20:1089-1091. doi:10.1038/cr.2010.124; published online 31 August 2010
}

Dendritic cells (DCs) are professional antigen-acquiring, -processing and -presenting cells [1-4]. As such, DCs form the key link between the innate and acquired immune responses playing a role in host defence and in immune tolerance [1-4]. Accordingly, defects in the ability of DCs to function can lead to increased susceptibility to infection, loss of tolerance, autoimmunity and tumour growth [1-4]. Sub-classes of DCs are defined and discriminated by the expression of different cell surface markers. Identification of factors that affect the activation or function of DCs is important in increasing our understanding of how these cells are regulated physiologically and of how they may become impaired leading to pathological conditions. Furthermore, knowledge of these factors and of their mechanisms of action may aid the design of strategies aimed at avoiding or repairing the consequences of poor DC function.

Many different lipids, including fatty acids, phospholipids, sphingolipids, sterols, lipoproteins, their breakdown products, and their oxidation products produced either enzymatically or nonenzymatically, have been demonstrated to influence immune cell function, although their effect on DCs have not

Correspondence: Philip C Calder

E-mail: pcc@soton.ac.uk been intensively studied. However, in the last few years a series of papers have appeared focusing on the impact of specific fatty acids on DC function. Loscher et al. [5] showed that conjugated linoleic acid suppresses nuclear factor (NF) $\kappa B$ activation and interleukin (IL)12 production in murine bone marrowderived DCs and acts through induction of IL-10 mediated by ERK. Weatherill et al. [6] reported that several saturated fatty acids directly activate murine bone marrow-derived DCs via toll-like receptor (TLR) 4 engagement leading to upregulation of several co-stimulatory molecules and pro-inflammatory cytokines. They also demonstrated that these effects could all be antagonized by the omega-3 fatty acid docosahexaenoic acid (DHA). DHA also inhibited the pro-inflammatory effects of bacterial lipopolysaccharide (LPS) on DCs. Zeyda et al. [7] showed that both the omega-3 fatty acid eicosapentaenoic acid (EPA) and the omega- 6 fatty acid arachidonic acid (ARA) inhibited LPS-induced upregulation of several co-stimulatory molecules and pro-infammatory cytokines like IL-12 and tumor necrosis factor- $\alpha$ (TNF- $\alpha)$ and impaired the ability of these DCs to stimulate allogeneic T cells. The effects of EPA and ARA on co-stimulatory molecules and cytokines were seen at the level of gene expression but did not involve an effect on ERK or JNK or on the NFKB system. Wang et al. [8] confirmed the inhibitory effects of EPA and DHA on co-stimulatory molecule expression, IL-12 and TNF- $\alpha$ gene expression and production, and $\mathrm{T}$ cell stimulation by human monocyte-derived DCs. Furthermore, EPA and DHA were found to suppress the phosphorylation, and so the activity, of p38 MAPK [8], suggesting an effect of the fatty acids on early signaling events in DCs. The authors speculated about the possible involvement of TLR4 upstream and NFKB downstream in the fatty acid effects they observed. Zapata-Gonzalez et al. [9] also confirmed the effects of DHA on co-stimulatory molecule expression, cytokine production and $\mathrm{T}$ cell stimulation by human monocyte-derived DCs and they demonstrated that EPA, the omega- 6 fatty acid linoleic acid and the monounsaturated fatty acid oleic acid had similar effects to DHA but were weaker in activity. Importantly, these authors showed that DHA had actions rather like peroxisome proliferator activated receptor (PPAR) $\gamma$ agonists and indeed the effects of DHA on DCs were blocked by an inhibitor of PPAR $\gamma$ [9]. PPAR $\gamma$ is expressed in DCs and blocks induction of pro-inflammatory responses. DHA-derived metabolites have been shown to act as PPAR $\gamma$ ligands and activators [10]. Furthermore, while PPAR $\gamma$ directly regulates inflammatory gene expression, it also interferes with the activation of $\mathrm{NF} \kappa \mathrm{B}$ creating an intriguing interaction between these 
two transcription factors [11]. Most recently, Kong et al. [12] confirmed lower co-stimulatory molecule expression, and TLR-induced pro-inflammatory cytokine and chemokine production by murine bone marrow-derived DCs exposed to DHA. DHA was shown to enhance PPAR $\gamma$ activity and reduce $\mathrm{NF \kappa B}$ activation. However, the effect of DHA on NFkB appeared to be PPAR $\gamma$ independent and to involve inhibition of I $\mathrm{B}$ degradation [12]. Thus, the picture that emerges from this series of reports is that several fatty acids may have a crucial role in regulating DC maturation and function and that fatty acids most likely act within signaling pathways with possible actions on membrane receptors (TLR), cytosolic protein kinases, and nuclear transcription factors (PPAR $\gamma, \mathrm{NF \kappa B}$ ). These actions may be linked by a single unifying mechanism or by two (or more) parallel mechanisms which may also involve membrane rafts and lipid mediator generation by DCs [13].

A report published in the August issue of Nature Medicine has suggested a novel and intriguing mechanism by which exogenous lipids can affect DC function [14]. In this study DCs from several sites in tumor-bearing mice were seen to accumulate intracellular lipid mainly in the form of triglyceride. Furthermore, DCs from patients with various cancers also showed lipid accumulation. In vitro studies with murine bone marrow-derived DCs showed that one or more substances released from tumors induced lipid (i.e. triglyceride) accumulation. Uptake of the saturated fatty acid palmitic acid into DCs was also increased by exposure of DCs to tumor conditioned medium, suggesting that the lipid accumulation may be driven by enhanced cellular uptake of fatty acids (and other lipids) and their subsequent storage. An examination of expression of four scavenger receptors revealed that one of these, macrophage scavenger receptor A (SRA) or CD204, was enhanced by exposure of DCs to the tumor conditioned medium. Furthermore, DCs from tumor-bearing mice that had accumulated high levels of intracellular lipid expressed higher levels of SRA. Finally lipid accumulation by cultured DCs was prevented by blocking scavenger receptors or by an antibody to SRA and did not occur for DCs from SRA knockout mice. Thus, SRA appears to be involved in tumorassociated lipid accumulation by DCs in vivo and in vitro. Rather little is known about the mechanism of fatty acid uptake into immune cells, including DCs, and SRA is certainly not fatty acid, or even lipid, specific. It would have been interesting to examine whether blocking or absence of SRA prevented lipid accumulation driven by exposure to palmitic acid rather than to the tumor conditioned medium and to investigate expression of membrane fatty acid transporters in DCs [15]. DCs that had accumulated lipid either in vivo or in vitro had impaired $\mathrm{T}$ cell stimulatory activity which was related to the reduced ability to process and present antigen. It is not clear how the accumulated lipid interferes with antigen handling in DCs. The final experiments presented by Herber $e t a l$. [14] involve the use of an acetyl-CoA carboxylase inhibitor. Acetyl-CoA carboxylase catalyses the conversion of acetyl-CoA to malonyl-CoA, the rate limiting step in fatty acid synthesis. Typically this process uses acetyl-CoA generated from glucose metabolism, but recycling of acetyl-CoA from fatty acid $\beta$-oxidation and from elsewhere is also a possible source of substrate. The inhibitor prevented accumulation of "lipids", clearly shown elsewhere to be triglyceride, in DCs upon exposure to the tumor conditioned medium. This curious finding is never explained by the authors, but suggests one of three possibilities. The first is that extracellular lipids being taken up via SRA (and other lipid uptake mechanisms) need to be degraded to the level of acetyl-CoA before resynthesis of fatty acids and their subsequent incorporation into triglyceride which is then accumulated. An experiment examining the effect of palmitic acid in the presence of the acetyl-CoA inhibitor would have been enlightening in this regard. The second possibility is that the accumulated lipid is derived from glucose and that the process of lipid accumulation from glucose via acetyl-CoA is driven by a factor present in the tumor conditioned medium. This possibility is not entirely compatible with the effects of palmitic acid and of blocking SRA. The third possibility is that the inhibitor sequesters coenzyme $\mathrm{A}$, since it is capable of forming a CoA ester itself, and that this disrupts the ability of triglycerides to form, since this requires fatty acyl-CoA to be synthesized as the fatty acid donor. Thus, the situation of triglyceride hydrolysis and inability to reform triglycerides would, over time, favor intracellular triglyceride loss.

These new findings [14] suggest a novel mechanism by which fatty acids and other lipids might affect DC function. There are a number of physiologic and pathologic conditions involving a state of hyperlipidaemia, including many cancers, diabetes, obesity, atherosclerosis, pregnancy and the response to injury. The findings presented by Herber et al. [14] show that there is a link between tumor bearing and lipid accumulation in DCs which in turn is related to an impairment of DC function that may be of significance in the host-tumor battle. It will be important to see if DC lipid accumulation and subsequent loss of function is a generalizable phenomenon that occurs across other hyperlipidaemic conditions, or whether it is tumor-derived factors that drive the process marking this out as a cancer-specific concern. Thus, whether lipid accumulation by DCs is a central mechanism to explain the immune impairments that occur in the above conditions remains to be seen. Although it is not clear how lipid accumulation might impair DC function, such accumulation seems to be exclusive from the 
mechanisms outlined in earlier work in the field of fatty acids and DC function [5-12]. Nevertheless, given the different reported effects of various fatty acids on DC responses [5-12], it would be of interest to ascertain whether exposure to different fatty acids, either alone or in combination, can influence lipid accumulation in DCs and the functional consequences.

The lipid accumulated in DCs as a result of their exposure to tumor conditioned medium [14] has a similar appearance under the electron microscope to so-called "lipid droplets" $[16,17]$. Lipid droplets, also known as lipid bodies, have been described in many cell types, including immune cells. Lipid droplets form upon immune stimulation and they have been described to appear or to become larger and more evident during infection and in inflammatory conditions $[16,17]$. They seem to play a role in the generation of eicosanoids which are often pro-inflammatory and immunosuppressive and, interestingly, the formation of lipid droplets is inhibited by a fatty acid synthesis inhibitor [17]. Clearly intracellular accumulation of lipid, most likely in structured droplets, is a routine response of cells, including immune cells, to certain environments. It will be important to identify the mechanism of lipid accumulation and the precise source of the lipid stored, how lipid accumulation impairs DC function, strategies to modulate lipid accumulation, and how lipid accumulation relates to and links with membrane, cytosolic and nuclear sites of action of fatty acids and other lipids on DCs.

\section{References}

1 Coquerelle C, Moser M. DC subsets in positive and negative regulation of immunity. Immunol Rev 2010; 234:317334.

2 Tisch R. Immunogenic versus tolerogenic dendritic cells: a matter of maturation. Int Rev Immunol 2010; 29:111118.

3 Swiecki M, Colonna M. Unraveling the functions of plasmacytoid dendritic cells during viral infections, autoimmunity, and tolerance. Immunol Rev 2010; 234:142-162

4 Ilett EJ, Prestwich RJ, Melcher AA. The evolving role of dendritic cells in cancer therapy. Expert Opin Biol Ther 2010; 10:369-379.

5 Loscher CE, Draper E, Leavy O, Kelleher D, Mills KH, Roche HM. Conjugated linoleic acid suppresses NF-kappa $\mathrm{B}$ activation and IL-12 production in dendritic cells through ERK-mediated IL-10 induction. J Immunol 2005; 175:4990-4998.

6 Weatherill AR, Lee JY, Zhao L, Lemay DG, Youn HS, Hwang DH. Saturated and polyunsaturated fatty acids reciprocally modulate dendritic cell functions mediated through TLR4. J Immunol 2005; 174:5390-5397.

7 Zeyda M, Säemann MD, Stuhlmeier $\mathrm{KM}$, et al. Polyunsaturated fatty acids block dendritic cell activation and function independently of NF-kappaB activation. J Biol Chem 2005; 280:1429314301.

8 Wang H, Hao Q, Li QR, et al. Omega-3 polyunsaturated fatty acids affect lipopolysaccharide-induced maturation of dendritic cells through mitogen-activated protein kinases p38. Nutrition 2007; 23:474-482.

9 Zapata-Gonzalez F, Rueda F, Petriz J, et al. Human dendritic cell activities are modulated by the omega- 3 fatty acid, docosahexaenoic acid, mainly through PPAR(gamma):RXR heterodimers: comparison with other polyunsaturated fatty acids. J Leukoc Biol 2008; 84:1172-1182.

10 Yamamoto $\mathrm{K}$, Itoh $\mathrm{T}$, Abe $\mathrm{D}$, et al. Identification of putative metabolites of docosahexaenoic acid as potent PPARgamma agonists and antidiabetic agents. Bioorg Med Chem Lett 2005; 15:517-522.

11 Van den Berghe W, Vermeulen L, Delerive P, De Bosscher K, Staels B, Haegeman G. A paradigm for gene regulation: inflammation, NF-kappaB and PPAR. Adv Exp Med Biol 2003; 544:181-196.

12 Kong W, Yen JH, Vassiliou E, Adhikary $\mathrm{S}$, Toscano MG, Ganea D. Docosahexaenoic acid prevents dendritic cell maturation and in vitro and in vivo expression of the IL-12 cytokine family. Lipids Health Dis 2010; 9:12.

13 Calder PC. The relationship between the fatty acid composition of immune cells and their function. Prost Leukotr Essent Fatty Acids 2008; 79:101-108.

14 Herber DL, Cao W, Nefedova Y, et al. Lipid accumulation and dendritic cell dysfunction in cancer. Nat Med 2010; 16:880-886.

15 Schwenk RW, Holloway GP, Luiken JJ, Bonen A, Glatz JF. Fatty acid transport across the cell membrane: regulation by fatty acid transporters. Prost Leukot Essent Fatty Acids 2010; 82:149-154.

16 D’Avila H, Maya-Monteiro CM, Bozza PT. Lipid bodies in innate immune response to bacterial and parasite infections. Int Immunopharmacol 2008; 8:1308-1315.

17 Bozza PT, Viola JP. Lipid droplets in inflammation and cancer. Prost Leukot Essent Fatty Acids 2010; 82:243-250. 